\title{
Essais de dosimètres neutrons à bulles, modèle BD 100 R-PND et modèle BDT
}

\author{
M. CHEMTOB*, R. DOLLO**, C. COQUEMA*, J. CHARY*, C. GINISTY*
}

(Manuscrit reçu le 26 octobre 1994, révisé le 15 décembre 1994, accepté le 19 janvier 1995)

\begin{abstract}
RÉSUMÉ Après avoir rappelé les missions du Centre technique d'homologation de l'instrumentation de radioprotection (CTHIR) et le rôle du groupe de travail "Dosimétrie individuelle des neutrons", l'article décrit la procédure d'évaluation des performances en prenant pour exemple les dosimètres neutrons à bulles, type BD 100 R-PND et BDT. Ces appareils portés sur le tronc assurent une dosimétrie individuelle. Les grandeurs de référence auxquelles ces dosimètres sont soumis sont celles des rapports ICRU 39 et 47 . Les conditions et les résultats des essais sont donnés. Il en ressort que ces appareils ont des performances suffisantes et conformes aux données du constructeur. Cependant, leur exploitation requiert des recommandations d'emploi.
\end{abstract}

ABSTRACT After having recalled the missions of the "Centre technique d'homologation de l'instrumentation de radioprotection (CTHIR)" and the function of the working group on personal neutron dosimetry, the performance evaluation procedure is described, taking as an example the bubble neutron dosimeters, type BD $100 \mathrm{R}$ PND and BDT. These instruments worn on the trunk allow personal dosimetry. The reference quantities to which these dosimeters are subjected are those of ICRU 39 and 47. Test conditions and results are given. It is concluded that these instruments have sufficient performances which comply with the data stated by the manufacturer. However, their exploitation requires recommendations of use.

\section{Introduction}

Le Centre technique d'homologation de l'instrumentation de radioprotection (CTHIR) a pour mission d'évaluer, par des essais et des comparaisons aux normes en vigueur, les performances des matériels destinés à la surveillance radiologique des personnes, des installations et de l'environnement. Pour ce faire, il effectue des essais selon des procédures normalisées, dans des laboratoires agréés. Les résultats des essais sont comparés aux normes ou autres documents contractuels (cahier des charges ou notices techniques). De plus, il

Institut de protection et de sûreté nucléaire, Département de protection de l'environnement et des installations, Service technique d'équipements de sureté et de radioprotection, Centre technique d'homologation de l'instrumentation de radioprotection. BP 6, F 92265 Fontenay-aux-roses Cedex.

** Électricité de France, Exploitation du parc nucléaire (EDF-EPN), mission Radioprotection, 92060 Paris la Défense Cedex 57. 
établit des recommandations d'emploi représentant des conseils de bon usage. Depuis sa création en 1982, le CTHIR a publié 63 documents d'évaluation d'instruments à la demande d'organismes constructeurs ou utilisateurs.

A l'heure actuelle, la dosimétrie des personnels travaillant dans des champs de rayonnements neutroniques est un problème encore mal résolu. Cette dosimétrie est essentiellement fondée sur la détection des particules secondaires engendrées par les neutrons dans des matériaux cibles (effet de chaque interaction avec le milieu) ou par l'analyse des modifications des propriétés physiques des matériaux détecteurs. L'ensemble de ces dosimètres passifs présente l'avantage d'une bonne fiabilité mais nécessite, pour la plupart, un étalonnage à chaque poste de travail. De plus, l'association de plusieurs types de dosimètres est souvent nécessaire pour couvrir la totalité de la plage d'énergie des neutrons.

Cette situation a amené EDF à créer, en 1992, un groupe de travail, en relation avec le Comité de radioprotection opérationnelle, réunissant les divers exploitants français concernés par la dosimétrie des neutrons (CEA, COGEMA, MELOX, EDF et, plus tard, la Délégation générale à l'armement (DGA) et l'IPSN, avec le double objectif suivant :

- à court terme (1993-1994) : définir les précautions et limites d'utilisation des seuls dosimètres individuels à lecture directe disponibles actuellement, à savoir les dosimètres à bulles (objet du rapport d'essais rédigé par le CTHIR) ;

- à moyen terme $(1996 \ldots)$ : choisir un dosimètre évolué à lecture directe capable de générer des alarmes en dose et débit d'équivalent de dose en local, grâce à un appel d'offre international reposant sur un cahier des charges exhaustif.

Pour l'action à moyen terme, un cahier des charges commun, établi par les exploitants, a été envoyé à une dizaine de constructeurs en mars 1993. La mise au point d'un dosimètre individuel électronique actif est liée à la résolution de la principale difficulté due à la réinjection du comptage des impulsions issues des photons gamma assimilables à celles produites par les neutrons de faible énergie. La dosimétrie des neutrons exige encore beaucoup d'efforts pour parvenir à une meilleure rigueur scientifique. Actuellement, les exploitants ont défini leur demande technique commune, avec le soutien actif d'ingénieurs du Service de dosimétrie (SDOS) de l'IPSN, auprès des constructeurs pour parvenir à la fabrication d'un dosimètre électronique.

Compte tenu du faible marché industriel ( 3000 dosimètres) et de la nécessité d'améliorer la performance technique, se pose la question de poursuivre le développement du dosimètre électronique (qui fera l'objet d'un prochain article).

Cet article prend pour exemple les dosimètres à bulles pour montrer la façon dont est conduite l'évaluation des performances. 
Les appareils en essais sont :

- le dosimètre neutrons à bulles, modèle BD 100 R-PND,

- le dosimètre neutrons à bulles, modèle BDT.

Ces appareils exposés aux neutrons sont le lieu de bulles dont le nombre est proportionnel à la dose ou à l'équivalent de dose subis.

Ils sont essentiellement constitués :

- d'une éprouvette en plastique obturée de manière étanche et contenant un polymère élastique dans lequel sont dispersées des microgouttelettes de liquide surchauffé ;

- d'une chambre à décompression et recompression surmontant ce polymère, cette chambre fonctionne à l'aide d'un piston manœuvré par un système à vis moletée.

Le modèle $\mathrm{BD} 100 \mathrm{R}-\mathrm{PND}$, porté sur le tronc, est destiné à la dosimétrie individuelle des neutrons rapides d'énergie supérieure à $200 \mathrm{keV}$. Le modèle BDT, porté lui aussi sur le tronc, est destiné à la dosimétrie individuelle des neutrons thermiques et épithermiques. Le fabricant est "Bubble technology industries".

\section{Caractéristiques annoncées par le constructeur}

Les caractéristiques annoncées par le constructeur du modèle BD $100 \mathrm{R}$ PND sont les suivantes :

- réponse en énergie : variation comprise entre $\pm 10 \%$ pour des énergies de $200 \mathrm{keV}$ à $15 \mathrm{MeV}$;

- étendue de mesures en équivalent de dose ; $1 \mu \mathrm{Sv}$ à $0,01 \mathrm{~Sv}(0,1 \mathrm{mrem}$ à $1 \mathrm{rem})$

- sensibilité typique : de 0,33 à 33 bulles/mrem ${ }^{1}$; les valeurs de sensibilité disponibles sont typiquement $0,33,2,2$ et 27 bulles/mrem, les valeurs intermédiaires peuvent être demandées ;

- sensibilité aux $\gamma$ : le dosimètre est insensible aux $\gamma$;

- réponse angulaire : isotrope ;

- réutilisation possible par recompression;

- durée de vie effective : 3 mois s'il est activé par décompression chaque jour ;

1. Nous n'avons pas converti les unités de dose (ou de débit de dose) de millirem en millisievert par commodité. En effet, la lecture à l'œil de ce dosimètre ne peut s'effectuer commodément que si elle ne dépasse pas 20 bulles, soit, pour une sensibilité de 1 bulle $\cdot \mathrm{mrem}^{-1}$, un équivalent de dose ne dépassant pas $0,2 \mathrm{mSv}$ (20 mrem). 
- durée de stockage : 1 an à la température ambiante sans choc thermique ;

- gamme opérationnelle :

- température : 20 à $40{ }^{\circ} \mathrm{C}$ (variation de la sensibilité comprise entre \pm $15 \%)$;

- humidité relative : jusqu'à $100 \%$;

- dimensions : $19 \mathrm{~mm}$ de diamètre $\mathrm{x} 131 \mathrm{~mm}$ de longueur ;

- masse : $41 \mathrm{~g}$;

- accessoire : lecteur automatique de dosimètres, type BDR série II, incluant un système optique de vision et un micro-ordinateur type PC équipé d'un logiciel de comptage des bulles; pour des lectures inférieures à 10 ou 20 bulles, le dosimètre peut être lu par comptage visuel.

Le modèle BDT a les mêmes caractéristiques que le modèle BD $100 \mathrm{R}$ PND, sauf qu'il mesure, de par sa conception, les neutrons thermiques et épithermiques.

A la demande, le constructeur fournit des dosimètres avec une sensibilité exprimée en bulles par mrem. Cette sensibilité est vérifiée par irradiation, à l'aide d'une source de neutrons Am-Be. Elle est garantie à $\pm 20 \%$ pour une gamme de température comprise entre 20 et $40^{\circ} \mathrm{C}$. La conversion de la fluence de la source en équivalent de dose est basée sur le rapport $\operatorname{NCRP}^{\circ} 38$ [3].

\section{Principe}

Un liquide facilement vaporisable, c'est-à-dire ayant une faible chaleur latente de vaporisation, est inclus sous forme de micro-gouttelettes dans un polymère de grande viscosité. La tension de vapeur du liquide surchauffé est en équilibre métastable avec la tension superficielle du polymère. Cet équilibre peut être rompu par apport d'énergie extérieure, en particulier par une interaction entre une particule lourde chargée présentant un transfert linéique d'énergie suffisamment grand. Les particules lourdes chargées sont créées par irradiation neutronique. La composition du dosimètre est telle que le nombre de bulles formé est proportionnel à l'équivalent de dose (équivalent de dose du rapport NCRP $\mathrm{n}^{\circ} 38$ ).

Le dosimètre peut être remis à zéro par recompresssion, à l'aide du piston manœuvré par le bouchon à vis moletée. De ce fait, le dosimètre est réutilisable.

\section{Essais effectués et résultats obtenus}

Les laboratoires ayant participé à l'ensemble des essais sont les suivants :

- Etablissement technique central de l'armement, Centre d'études du Bouchet, Département "Détection-protection nucléaire", Service "Effets des 
rayonnements" (ETCA/.Arcueil), 16 bis, Avenue Prieur de la Côte d'or, 94114 Arcueil Cedex ;

- Département de protection de la santé de l'homme et de dosimétrie, Service de dosimétrie, Laboratoire d'études et de recherche en dosimétrie externe (DPHD/SDOS/LRDE, Fontenay-aux-Roses et Cadarache).

\subsection{Grandeurs d'étalonnage pour les essais}

Les grandeurs suivantes, de la catégorie de l'équivalent de dose, sont utilisées pour évaluer les performances des dosimètres :

- équivalent de dose ambiant, $H^{*}(10)$, pour les irradiations neutroniques effectuées dans l'air;

- équivalent de dose individuel, $H_{\mathrm{p}}(10, \alpha)$, pour les irradiations neutroniques effectuées avec les dosimètres placés sur et au centre de la face d'entrée du fantôme en PMMA (polyméthyl métacrylate de méthyl acétique) de $30 \mathrm{~cm}$ $\times 30 \mathrm{~cm} \times 15 \mathrm{~cm}$.

Les coefficients de conversion de la fluence neutronique en équivalent de dose ambiant sont tirés de la norme ISO 8529 [7]. Ceux relatifs à l'équivalent de dose individuel sont tirés d'un rapport PTB de Siebert et Schumacher (communication privée). Dans ces deux documents, la fonction reliant le facteur de qualité au transfert linéique d'énergie est celle figurant dans la publication ICRP 21 [4].

Du fait que ces appareils sont destinés à la dosimétrie individuelle, la grandeur qu'ils doivent mesurer est l'équivalent de dose individuel $H_{\mathrm{p}}(10, \alpha)$. Toutefois, dans ce document, pour les essais n'impliquant pas un étalonnage ou donnant des résultats en valeurs relatives, la grandeur de référence est l'équivalent de dose ambiant.

\section{Remarque}

Dans ce document:

- le terme sensibilité est utilisé quand la grandeur de référence n'est pas celle recommandée par les rapports ICRU 39 et 47 [5-6], pour la dosimétrie individuelle;

- le terme réponse est utilisé quand la grandeur d'étalonnage est l'équivalent de dose individuel $H_{\mathrm{p}}(10, \alpha)$.

Dans chaque paragraphe ci-dessous sera précisée la grandeur de référence pendant l'essai.

Le rapport d'essais $n^{\circ} 101$ [1] précise toutes les conditions des essais. Dans ce document, certaines conditions, les plus essentielles, sont rappelées.

\subsection{Essais de réception et de dispersion des dosimètres BD 100R-PND}

Le but de cet essai est d'évaluer, à l'aide d'irradiations aux neutrons d'une source $\mathrm{Am}-\mathrm{Be}$, le lot de dosimètres fourni par le constructeur. Il consiste à vérifier : 
1) que la sensibilité de chaque détecteur annoncée par le constructeur est comparable à celle mesurée par les laboratoires mentionnés au $\S 4$;

2) que l'écart type du lot est conforme aux données du constructeur.

La sensibilité mesurée en bulles/mrem, est exprimée par la relation :

$$
S_{\mathrm{m}}=\frac{N_{i}}{H^{*}(10)}
$$

où $N_{i}$ est le nombre de bulles ( $\approx 100$ bulles) enregistré par le dosimètre ayant été soumis à l'équivalent de dose ambiant $H^{*}(10)$ pour l'essai $i$.

La sensibilité mesurée moyenne, $\bar{S}_{\mathrm{m}}$, est calculée pour chaque dosimètre en tenant compte des 5 essais effectués. Elle est comprise entre 1,1 et 2,5 bulles/mrem.

Pour les 5 essais, les valeurs de l'écart-type relatif trouvé sont comprises entre $2,5 \%$ et $15 \%$ pour les deux lots de 20 dosimètres évaluées à l'ETCA et au SDOS/LRDE.

Pour comparer la sensibilité mesurée moyenne à celle annoncée par le constructeur, pour chaque dosimètre, le quotient $q$ est calculé :

$$
q=\frac{S_{\mathrm{c}}}{\bar{S}_{\mathrm{m}}}
$$

où $S_{\mathrm{c}}$ est la sensibilité annoncée par le constructeur évaluée selon l'équivalent de dose défini dans le document NCRP $n^{\circ} 38$ [3].

La figure 1 donne les valeurs de ce quotient $q$ pour les deux lots de 20 dosimètres modèle BD 100 R-PND évalués à l'ETCA (a) et au SDOS (b). Elle donne également la valeur moyenne $\bar{q}$ et l'écart type de chaque lot. La variabilité de chaque lot de 20 détecteurs mesurée par 2 fois l'écart type relatif sur le quotient $q$ est égale à $\pm 10 \%$.

\subsection{Essais en température des dosimètres BD 100R-PND}

Le but de ces essais, effectués avec une source Am-Be, est de déterminer la variation de la sensibilité des dosimètres en fonction de la température.

La sensibilité mesurée en bulles/mrem, est déterminée pour les 10 dosimètres essayés aux températures $15,20,25,30,35$ et $40{ }^{\circ} \mathrm{C}$. L'essai pour chaque température est répété 3 fois. La sensibilité mesurée moyenne est calculée pour chaque dosimètre en prenant en compte les 3 essais effectués.

La sensibilité mesurée moyenne en fonction de la température pour les 10 dosimètres est montrée sur la figure 2. La figure 3 donne, pour le lot de 10 dosimètres, le rapport de la sensibilité moyenne du lot à la température $t^{\circ} \mathrm{C}$ et de celle obtenue à $20^{\circ} \mathrm{C}\left(S(t) / S\left(20^{\circ} \mathrm{C}\right)\right.$. Elle donne également pour chaque température l'écart type du rapport $\left(S(t) / S\left(20^{\circ} \mathrm{C}\right)\right.$. 


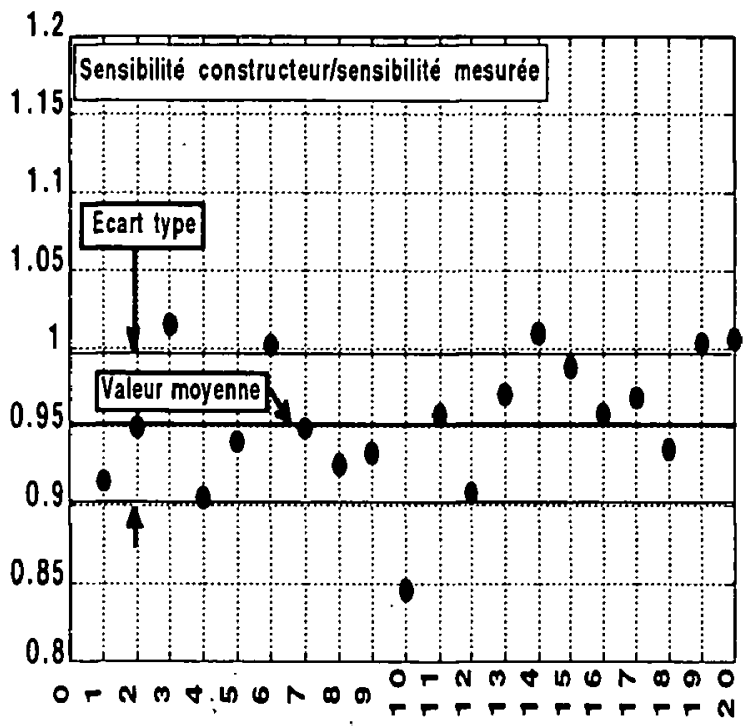

N'Détecteur

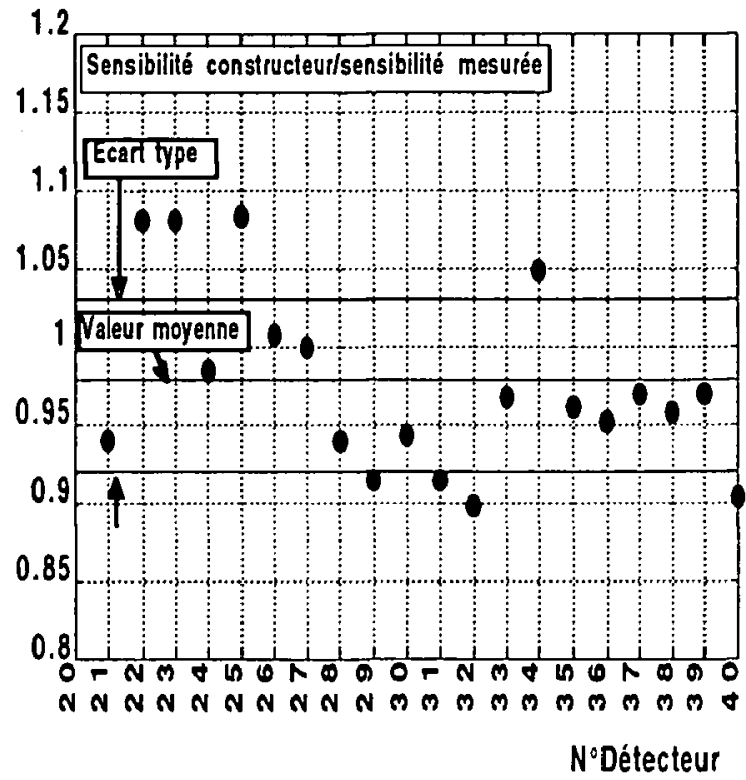

b

Fig. 1 - Dispersion de la sensibilité par rapport à celle du constructeur (ECTA) (a) et (SDOS) (b).

Sensitivity dispersion stated by manufacturer vs dispersion measured by (a) $E T C A$, (b) SDOS.
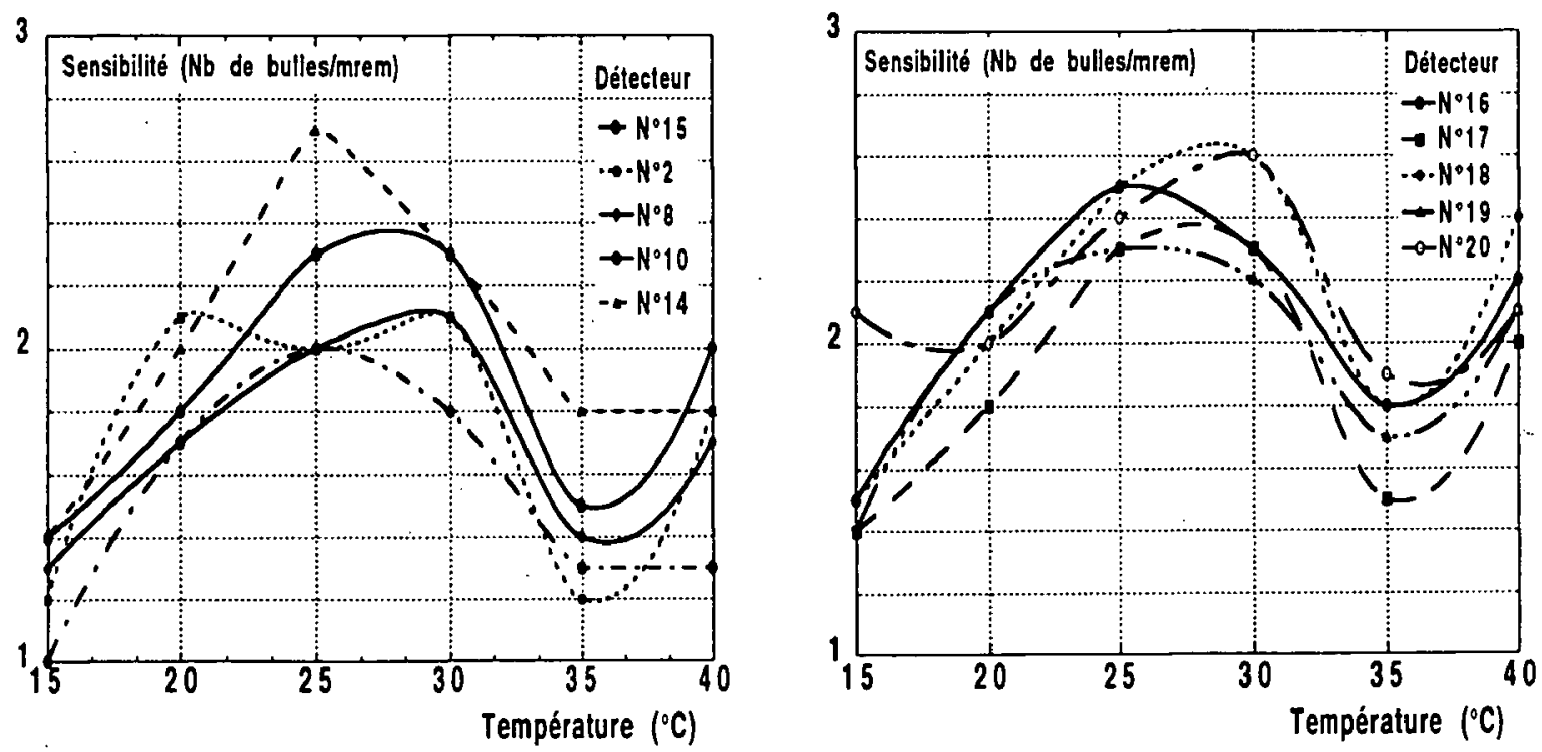

Fig. 2 - Sensibilité en fonction de la température.

Sensitivity as a function of temperature.

La courbe de la figure 3 a été communiquée aux laboratoires impliqués dans les essais, afin d'utiliser ce rapport comme coefficient de correction pour ramener à $20^{\circ} \mathrm{C}$ les valeurs lues à la température ambiante. 


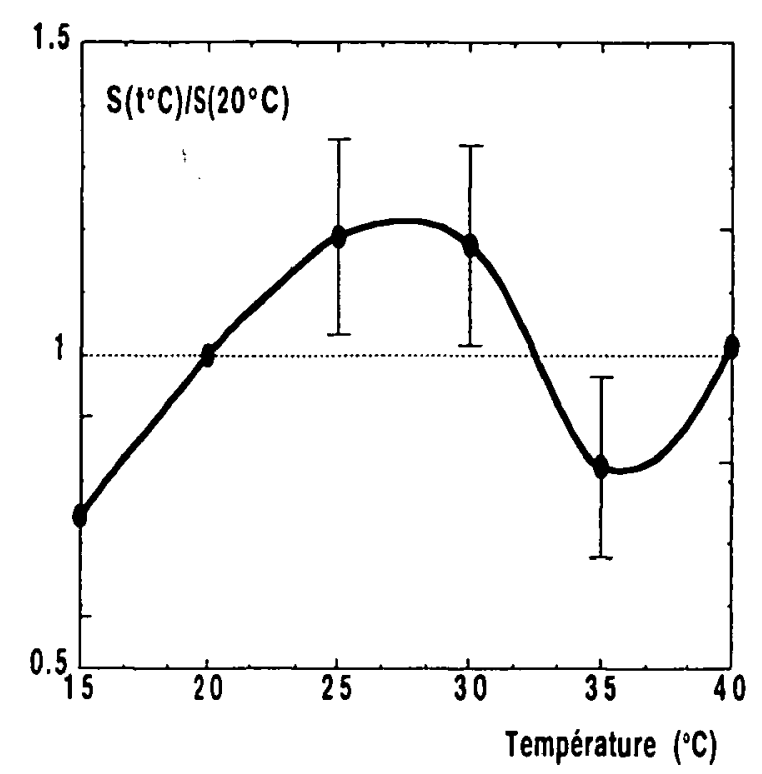

Fig. 3 - Coefficient de correction en fonction de la température. Correction coefficient as a function of temperature.

Les dosimètres ayant subi les essais en température ont été à nouveau irradiés et lus à $20^{\circ} \mathrm{C}$. La figure 4 donne la sensibilité à $20^{\circ} \mathrm{C}$ après chaque essai de température :

- en abscisse est portée la température $\left({ }^{\circ} \mathrm{C}\right)$ subie par le dosimètre ;

- en ordonnée est porté le rapport $\frac{S\left(t \rightarrow 20^{\circ} \mathrm{C}\right)}{S\left(20^{\circ} \mathrm{C}\right)}$ représentant la sensibilité relative du dosimètre après retour à $20^{\circ} \mathrm{C}$.
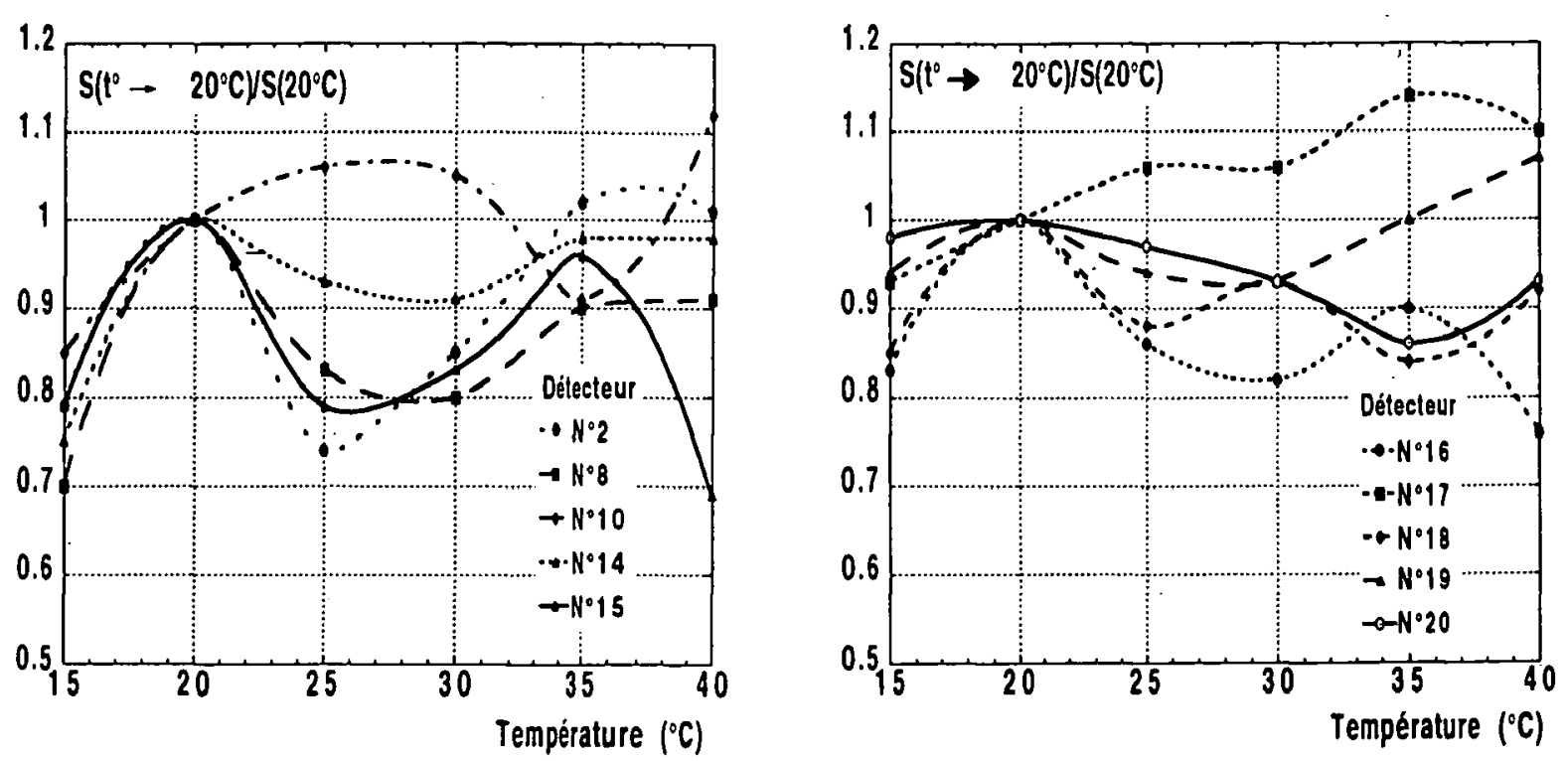

Fig. 4 - Sensibilité relative à $20^{\circ} \mathrm{C}$ après chaque essai de température.

Relative sensitivity at $20^{\circ} \mathrm{C}$ after each temperature test. 
La sensibilité relative moyenne du lot des 10 dosimètres à $20^{\circ} \mathrm{C}$ après les essais de température est montrée sur la figure 5.

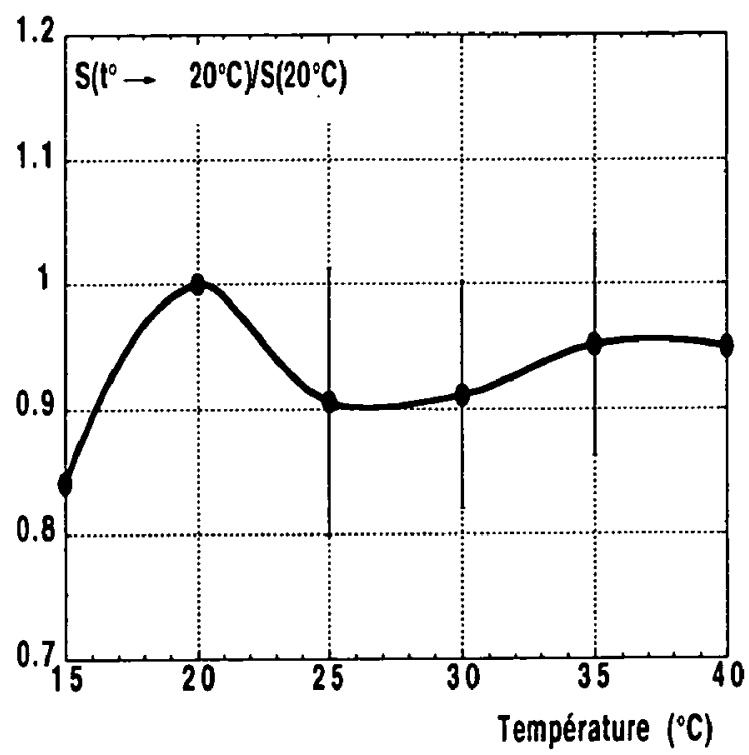

Fig. 5 - Sensibilité relative moyenne à $20^{\circ} \mathrm{C}$ après chaque essai de température. Mean relative sensitivity at $20^{\circ} \mathrm{C}$ after each temperature test

\subsection{Réponse des dosimètres BD 100R-PND}

en fonction du débit d'équivalent de dose individuel $H_{\mathrm{p}}(10, \alpha=0)$

Le but de cet essai est de vérifier que la réponse du dosimètre à un équivalent de dose donné $H_{\mathrm{p}}(10, \alpha=0)$, reste constante pour différents débits de cette grandeur. De plus, il donne un étalonnage du dosimètre dans le faisceau de référence des neutrons du ${ }^{252} \mathrm{Cf}$ et dans la grandeur $H_{\mathrm{p}}(10, \alpha=0)$.

La réponse en fonction du débit d'équivalent de dose individuel $\dot{H}_{\mathrm{p}}(10$, $\alpha=0$ ) est exprimée par la relation :

$$
R\left[\dot{H}_{\mathrm{p}}(10, \alpha=0)\right]=\frac{\bar{N}}{H_{\mathrm{p}}(10, \alpha=0)}
$$

où $\bar{N}$ est le nombre moyen de bulles compté dans le dosimètre subissant l'équivalent de dose $H_{\mathrm{p}}(10, \alpha=0)$ à un débit $\dot{H}_{\mathrm{p}}(10, \alpha=0)$, en tenant compte des 3 essais effectués.

L'écart type sur le nombre de bulles compté dans le dosimètre est calculé en prenant en compte les 3 essais effectués.

La réponse relative en fonction du débit d'équivalent de dose individuel est calculée, pour chaque dosimètre, selon la relation :

$$
R^{\prime}\left[\dot{H}_{\mathrm{p}}(10, \alpha=0)\right]=\frac{R\left[\dot{H}_{\mathrm{p}}(10, \alpha=0)\right]}{R\left[\dot{H}_{\mathrm{p}}(10, \alpha=0)=0,048 \mathrm{mSv} \cdot \mathrm{h}^{-1}\right]}
$$


La réponse normalisée, pour un dosimètre présentant une sensibilité (Am$\mathrm{Be})$ égale à 1 bulle/mrem, est calculée selon la relation :

$$
R_{\mathrm{n}}\left[H_{\mathrm{p}}(10, \alpha=0)\right]=\frac{R\left[H_{\mathrm{p}}(10, \alpha=0)\right]}{\overline{S_{\mathrm{m}}}}
$$

La figure 6 donne la réponse relative $R^{\prime}\left[\dot{H}_{\mathrm{p}}(10, \alpha=0)\right]$ normalisée par rapport au débit le plus faible $\left(48,2 \mu \mathrm{Sv} . \mathrm{h}^{-1}, 4,82 \mathrm{mrem} \cdot \mathrm{h}^{-1}\right)$.

La figure 7 donne la réponse relative moyenne $\bar{R}^{\prime}\left[\dot{H}_{\mathrm{p}}(10, \alpha=0)\right]$ et son écart type pour les 5 dosimètres.

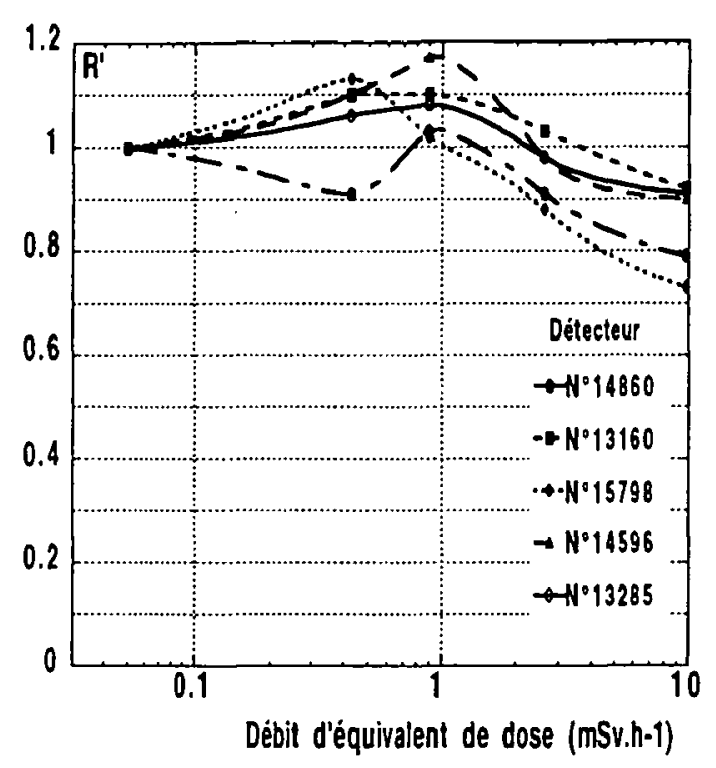

Fig. 6 - Réponse relative en fonction du débit d'équivalent de dose individuel. Relative response as a function of the individual dose equivalent rate.

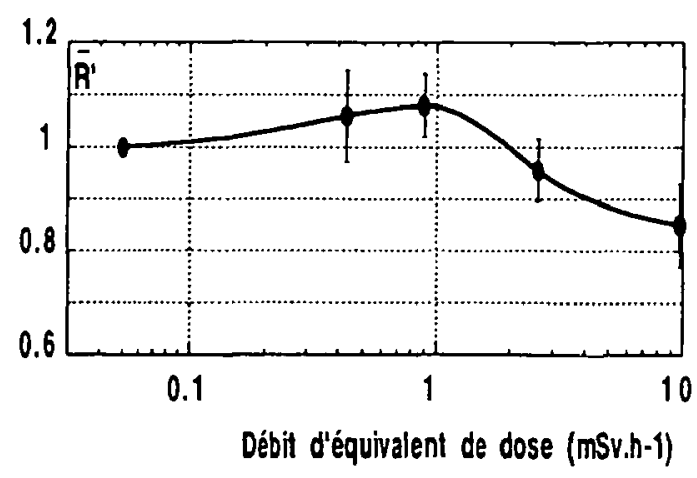

Fig. 7 - Réponse relative moyenne en fonction du débit d'équivalent de dose individuel. Mean relative response as a function of the individual dose equivalent rate. 


\section{Nota}

Réponses moyennes des 5 dosimètres, entre 5 et 262 mrem.h - $^{-1}(0,05$ $2,62 m S v)$ :

$\overline{\mathrm{R}}\left[\dot{H}_{\mathrm{p}}(10, \alpha=0)\right]=2,60$ avec un écart-type de 0,18 bulles $/ \mathrm{mrem}$

$\overline{\mathrm{R}}_{n}\left[\dot{H}_{\mathrm{p}}(10, \alpha=0)\right]=1,41$ avec un écart-type de 0,15 bulles $/$ mrem

\subsection{Réponse des dosimètres BD 100R PND en fonction de l'énergie des neutrons}

Le but de cet essai consiste à vérifier à partir de quelle énergie des neutrons le dosimètre commence à compter des bulles et à s'assurer que le nombre de bulles par unité d'équivalent de dose individuel, $H_{\mathrm{p}}(10, \alpha=0)$, reste constant dans des limites spécifiées, pour différentes énergies. Les neutrons monocinétiques sont obtenus à l'aide des accélérateurs Van de Graaf de BIII (Service de physique, CEN Bruyères-le-Châtel) et SAMES (SDOS/LRDE/CE-Cadarache).

La réponse en fonction de l'énergie est exprimée par la relation :

$$
R(E)=\frac{\bar{N}}{H_{\mathrm{p}}(10, \alpha=0)}
$$

où $\bar{N}$ est le nombre moyen de bulles compté dans le dosimètre subissant l'équivalent de dose $H_{\mathrm{p}}(10, \alpha=0)$ à l'énergie $E$, en tenant compte des 3 essais effectués.

L'écart type sur le nombre de bulles compté dans le dosimètre est calculé en tenant compte des 3 essais effectués. Il est compris entre 2 et $12 \%$, sauf aux énergies de 0,144 et $0,200 \mathrm{MeV}$ où il n'a pas été calculé à cause de la faible sensibilité du dosimètre à ces énergies. La figure 8 donne la réponse $R(E)$ pour les 5 dosimètres essayés. La figure 9 donne la réponse moyenne $\bar{R}_{\mathrm{n}}(E)$ rame-

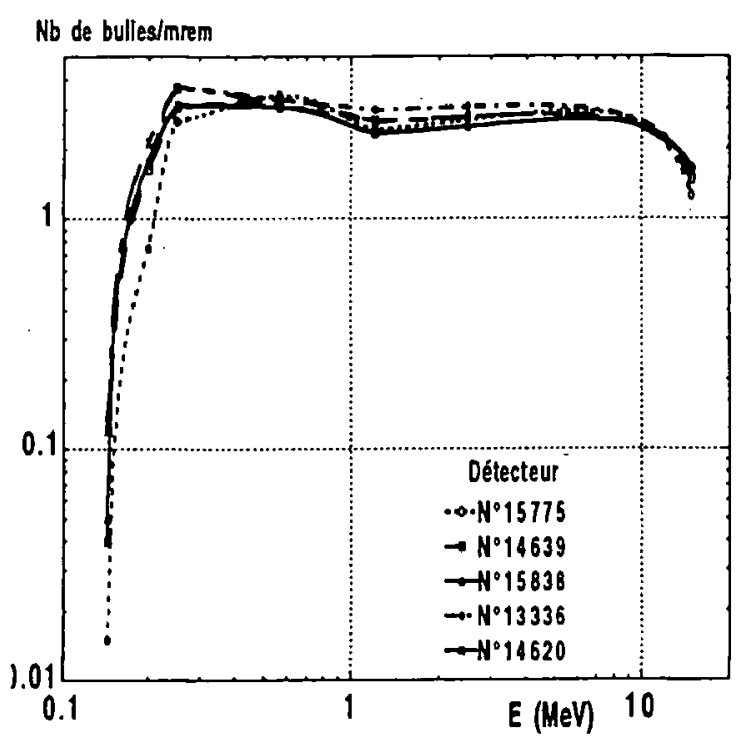

Fig. 8 - Réponse en fonction de l'énergie des neutrons $\left(H_{\mathrm{p}}(10, \alpha=0)\right)$.

Response as a function of neutron energy $\left(H_{p}(10, \alpha=0)\right.$ 


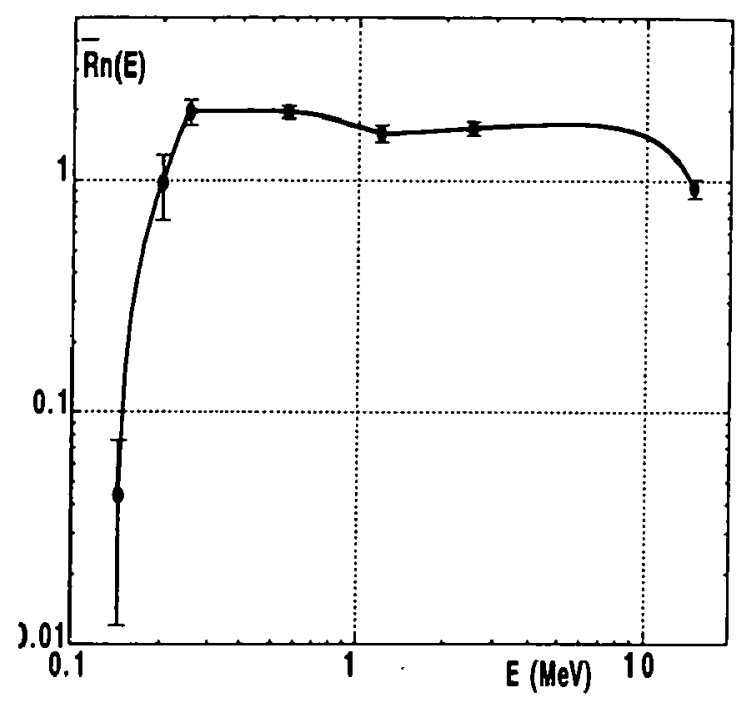

Fig. 9 - Réponse moyenne en fonction de l'énergie $\left(H_{\mathrm{p}}(10, \alpha=0)\right)$ normalisée pour une sensibilité Am-Be (1 bulle/mrem).

Mean response as a function of the energy $\left(\mathrm{H}_{\mathrm{p}}(10, \alpha=0)\right.$ standardised for AmBe sensitivity (1 bubble/mrem).

née à une sensibilité ( $\mathrm{Am}-\mathrm{Be})$ de 1 bulle/mrem et l'écart type de cette réponse, pour les 5 dosimètres.

\subsection{Réponse des dosimètres BD 100R-PND en fonction de l'angle d'incidence}

Le but de cet essai, effectué avec une source de ${ }^{252} \mathrm{Cf}$, est de vérifier que l'indication de ce dosimètre, placé sur le fantôme parallélépipédique, suit la grandeur équivalent de dose individuel en fonction de l'angle d'incidence. Les rotations $\alpha$ sur le plan horizontal (Fig. 10) sont effectuées avec un dosimètre placé sur et au centre de la face d'entrée du fantôme, autour du centre géométrique du dosimètre, par pas de $30^{\circ}$ entre $0^{\circ}$ et $\pm 90^{\circ}$. Les rotations $\theta$ sur le plan vertical (Fig. 10) sont effectuées avec 3 dosimètres disposés sur et au centre de la face d'entrée du fantôme, autour du centre géométrique du dosimètre central avec le pas de $30^{\circ}$ et entre $0^{\circ}$ et $\pm 90^{\circ}$.

Pour chaque dosimètre les réponses angulaires sur les deux plans sont calculées par les expressions :

$$
R(\alpha)=\frac{N(\alpha)}{N\left(\alpha=0^{\circ}\right)} \text { et } R(\theta)=\frac{N(\theta)}{N\left(\theta=0^{\circ}\right)}
$$

où $N(\alpha)$ est le nombre de bulles lu sur chaque dosimètre ayant été irradié à l'incidence $\alpha, N\left(\alpha=0^{\circ}\right)$ est celui enregistré à l'angle $\alpha=0^{\circ}$. Il en est de même pour $N(\theta)$ et $N\left(\theta=0^{\circ}\right)$.

Les figures $11 \mathrm{a}$ et $11 \mathrm{~b}$ donnent les réponses $R^{\prime}(\alpha), R^{\prime}(\theta)$ sur les plans précités et les limites de variations extraites du projet de la norme 


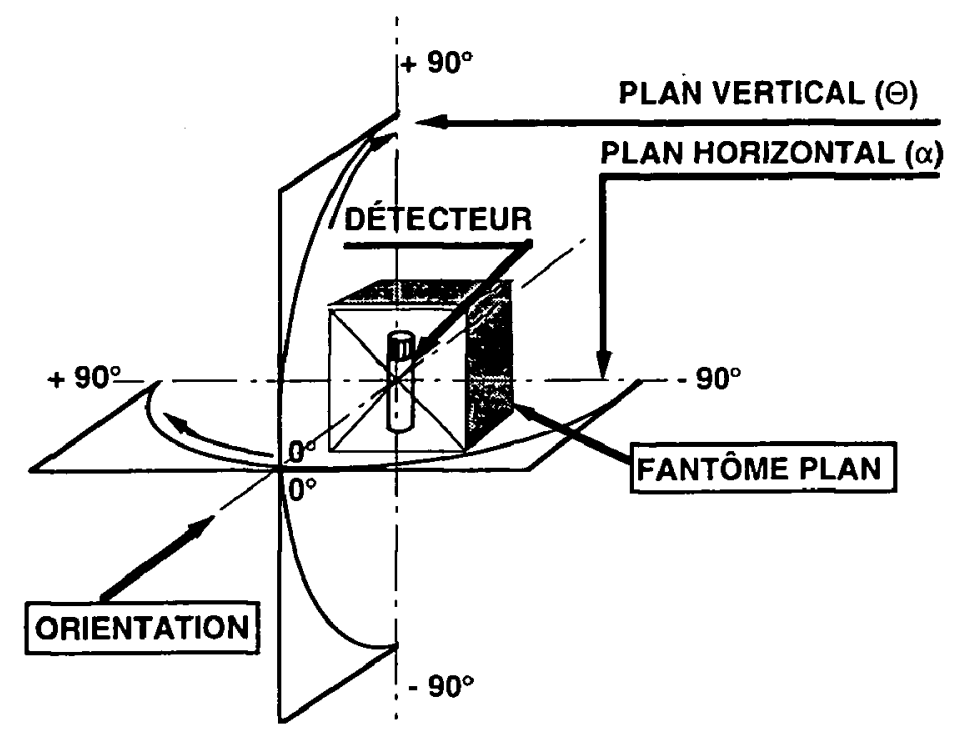

Fig. 10 - Réponse angulaire-plans de référence. Angular response-reference planes.

CEI 45B (Secrétariat) 120 [2] calculées en appliquant une tolérance de $\pm 30 \%$ au rapport $\frac{H_{\mathrm{p}}(10, \alpha)}{H_{\mathrm{p}}(10, \alpha=0)}$.

\subsection{Réponse des dosimètres BD 100R-PND à deux spectres différents}

Le but de cet essai est de déterminer la réponse du dosimètre à des spectres de neutrons polycinétiques réels :
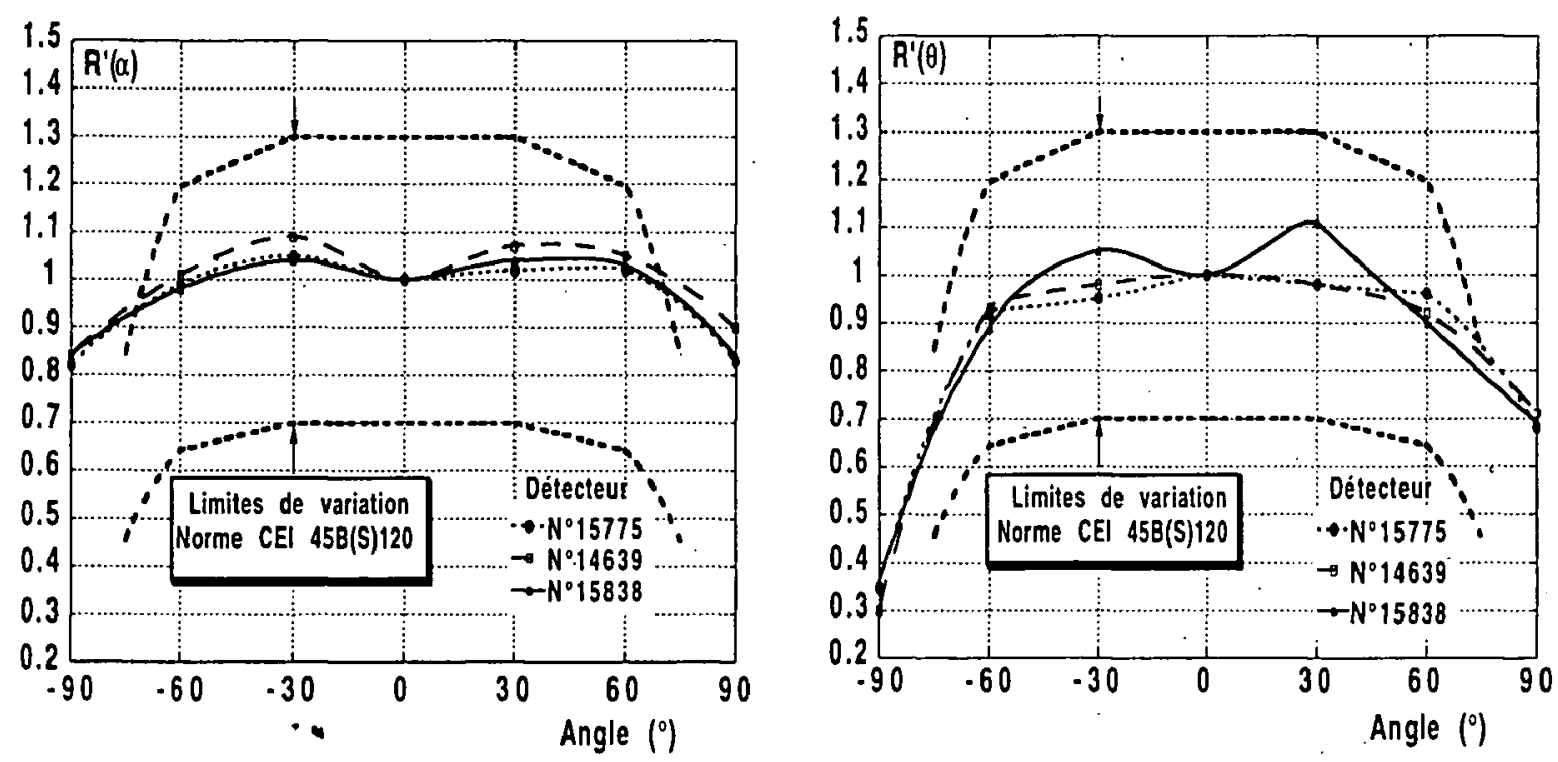

Fig. 11 - Réponse angulaire relative avec fantôme a) plan horizontal. b) plan vertical. Relative angular response with a phantom a) horizontal plane, b) vertical plane. 
- spectre CANEL comportant trois composantes, thermique, intermédiaire et rapide, d'équivalents de dose ambiants $H^{*}(10)$ respectivement égaux à $4 \%$, $2 \%$ et $94 \%$. L'équivalent de dose $H_{\mathrm{p}}(10, \alpha=0)$ auquel ont été soumis les dosimètres est égal à $1,35 \mathrm{mSv}(135 \mathrm{mrem})$;

- spectre CANEL $+\mathrm{H}_{2} \mathrm{O}$ (écran de $20 \mathrm{~cm}$ ) comportant les mêmes composantes, d'équivalent de dose ambiant $H^{*}(10)$ respectivement égaux à $14 \%, 5 \%$ et $81 \%$. L'équivalent $H_{\mathrm{p}}(10, \alpha=0)($ ICRP 21$)$ est, dans ce cas, égal à $1,30 \mathrm{mSv}$ (130 mrem).

La réponse de chaque dosimètre à ces spectres $R\left[H_{\mathrm{p}}(10, \alpha=0)\right]$ s'exprime par la relation :

$$
R\left[H_{\mathrm{p}}(10, \alpha=0)\right]=\frac{N}{H_{\mathrm{p}}(10, \alpha=0)}
$$

où $N$ est le nombre de bulles enregistré par chaque dosimètre.

$H_{\mathrm{p}}(10, \alpha=0)$ est l'équivalent de dose individuel auquel sont soumis les dosimètres.

La réponse moyenne normalisée à une sensibilité de 1 bulle/mrem (source Am-Be, voir $\S 4.2$ ) et son écart-type, pour les deux spectres, sont calculés en prenant en compte les 5 dosimètres :

1) spectre CANEL :

$\bar{R}_{\mathrm{n}}=0,55$ bulles/mrem

$s=0,15$ bulles/mrem

2) spectre CANEL $+\mathrm{H}_{2} \mathrm{O}$ (écran $20 \mathrm{~cm}$ )

$\bar{R}_{\mathrm{n}}=0,35$ bulles $/ \mathrm{mrem}$

$s=0,08$ bulles $/$ mrem

Il faut rappeler que la réponse $\bar{R}_{\mathrm{n}}$ aux neutrons du ${ }^{252} \mathrm{Cf}$, est de $1,41 \mathrm{bulles} / \mathrm{mrem}$ avec un écart-type de $0,15 \mathrm{bulles} / \mathrm{mrem}$.

Les écarts de réponse entre les spectres du ${ }^{252} \mathrm{Cf}$, CANEL et CANEL $+\mathrm{H}_{2} \mathrm{O}$ peuvent s'expliquer par une plus grande contribution, pour ces deux derniers, des neutrons thermiques et d'énergie inférieurs à $200 \mathrm{keV}$, à l'équivalent de dose. Pour cette raison, il est recommandé de doubler ce dosimètre par un deuxième du modèle BDT.

\subsection{Réponse et sensibilité aux neutrons thermiques du dosimètre BDT}

Le but de cet essai est d'étalonner le dosimètre BDT aux neutrons thermiques et de vérifier :

1) que la sensibilité de chaque détecteur annoncée par le constructeur est comparable à celle mesurée par les laboratoires mentionnés au $\$ 4$; 
2) que l'écart-type du lot est conforme aux données du constructeur.

Les caractéristiques essentielles de la source de neutrons thermiques du réacteur HARMONIE (Centre d'études de Cadarache) sont les suivantes :

- le débit de fluence des neutrons thermiques est déterminé par des détecteurs à activation à pastilles d'or et confirmé par des pastilles de cuivre; le débit de fluence retenu est celui de l'or, soit 4,65 $\times 104 \mathrm{n} \cdot \mathrm{cm}^{-2} \cdot \mathrm{s}^{-1}$; le débit de fluence des neutrons d'énergie supérieure à $0,51 \mathrm{eV}$ a été quantifié par une pastille d'or sous cadmium ;

- le spectre des neutrons est déterminé à l'aide de la méthode multisphère ; il est compris entre $1 \times 10^{-2}$ et $0,5 \mathrm{eV}$.

La réponse de chaque dosimètre, mesurée en bulles/mrem, est exprimée par la relation :

$$
R\left[H_{\mathrm{p}}(10, \alpha=0)\right]=\frac{N}{H_{\mathrm{p}}(10, \alpha=0)}
$$

Ce résultat représente l'étalonnage du dosimètre.

La sensibilité de chaque dosimètre, mesurée en bulles/mrem, est exprimée par la relation :

$$
S_{\mathrm{m}}=\frac{N}{H^{*}(10)}
$$

La sensibilité $S_{\mathrm{m}}$ est déterminée pour la comparer à celle $S_{\mathrm{c}}$ annoncée par le constructeur. lité.

Le tableau I donne les réponses des cinq dosimètres ainsi que leur sensibi-

\subsection{Sensibilité des dosimètres BD 100R-PND et BDT aux $\gamma$}

Le but de cet essai est de vérifier que ces dosimètres sont insensibles aux $\gamma$.

Dans aucun des détecteurs il n'a été constaté la présence de bulles, les dosimètres sont insensibles aux photons $\gamma$ du ${ }^{60} \mathrm{Co}$.

\section{Conclusion}

A partir des essais effectués et des résultats obtenus, il ressort que :

- les détecteurs BD 100R-PND livrés ont une sensibilité annoncée par le constructeur aux neutrons de la source $\mathrm{Am}-\mathrm{Be}$, exprimée dans la grandeur 
TABLEAU I

Réponse et sensibilité du dosimètre BDT aux neutrons thermiques BDT dosemeter response and sensitivity to thermal neutrons

\begin{tabular}{|c|c|c|c|c|c|}
\hline No dosimetre & 215 & 200 & 222 & 210 & 218 \\
\hline$R\left[H_{\mathrm{p}}(10, \alpha=0)\right]$ (bulles/mrem) & 5,05 & 5,46 & 5,09 & 4,95 & 5,25 \\
\hline$S_{\mathrm{m}}($ bulles/mrem $)$ & 3,76 & 3,76 & 4,03 & 4,16 & 3,80 \\
\hline$S_{\mathrm{c}}$ (bulles/mrem) & 3,0 & 3,3 & 3,2 & 3,2 & 3,3 \\
\hline $\mathrm{q}=\frac{S_{\mathrm{c}}}{S_{\mathrm{m}}}$ & 0,80 & 0,88 & 0,79 & 0,77 & 0,87 \\
\hline $\bar{q} \pm s_{\mathrm{q}}$ & & & $2 \pm 0$ & & . \\
\hline $\begin{array}{l}R_{\mathrm{n}}\left[H_{\mathrm{p}}(10, \alpha=0)\right] \\
\text { pour } S_{\mathrm{m}}=1 \text { bulle/mrem } \\
\quad(\text { bulles } / \mathrm{mrem})\end{array}$ & 1,34 & 1,45 & 1,26 & 1,19 & 1,38 \\
\hline $\bar{R}_{\mathrm{n}} \pm S_{R_{\mathrm{n}}}$ (bulles/mrem) & & & $2 \pm 0$ & & \\
\hline
\end{tabular}

équivalent de dose (NCRP 38), comparable à celle mesurée en termes de l'équivalent de dose ambiant $H^{*}(10)$ à mieux que $\pm 10 \%$; le constructeur livre chaque lot accompagné d'une feuille de mesure des sensibilités ;

- la réponse du dosimètre BD 100R-PND décroît faiblement en fonction du débit d'équivalent de dose individuel $H_{\mathrm{p}}(10, \alpha=0)$, d'environ $15 \%$ pour un débit de 0,01 Sv.h ${ }^{-1}\left(1\right.$ rem.h $\left.{ }^{-1}\right)$, ce fait peut être expliqué par gaspillage de l'énergie, pour la formation des bulles, quand sa concentration instantanée est forte ;

- la réponse de ce dosimètre en énergie est nulle jusqu'à $\approx 200 \mathrm{keV}$; elle augmente et atteint un plateau entre $250 \mathrm{keV}$ et $2,5 \mathrm{MeV}$ s'établissant à 3 bulles/mrem pour des dosimètres dont la sensiblité à l'Am-Be est comprise entre 1,5 et 1,6 bulles/mrem ; elle décroît à 1,5 bulles/mrem à $14,8 \mathrm{MeV}$; cette décroissance peut s'expliquer par la diminution du transfert linéique d'énergie moyen $(\overline{T L E})$ des particules lourdes chargées de forte énergie ;

- la réponse angulaire de ce dosimètre reste comprise dans les tolérances fixées par la norme CEI 45B (S)120 relative aux dosimètres individuels (Fig. 11a et 11b);

- le comportement de ce dosimètre en température entre $+15^{\circ} \mathrm{C}$ et $+40^{\circ} \mathrm{C}$ est conforme à celui annoncé par le constructeur, c'est-à-dire qu'il pré- 
sente une variation relative de la sensibilité, par rapport à celle obtenue à $20{ }^{\circ} \mathrm{C}$, de $\pm 20 \%$ (il faut signaler qu'après retour à la température de $+20^{\circ} \mathrm{C}$ les dosimètres ayant préalablement subi les températures précitées montrent une perte maximale de sensibilité de l'ordre de $15 \%$ );

- pour tenir compte de composantes à l'équivalent de dose individuel dues à des neutrons d'énergies inférieures à $200 \mathrm{keV}$, il faut munir l'agent d'un deuxième dosimètre du type BDT ; la sensibilité de ce dernier aux neutrons thermiques par rapport à celle du constructeur est de 1,22 avec un écart-type du lot (5 dosimètres) de $\pm 0,073$; sa réponse, en termes de la grandeur $H_{\mathrm{p}}$ $(10, \alpha=0)$, ramenée à un dosimètre dont la sensibilité mesurée est de $1 \mathrm{bulle} / \mathrm{mrem}$, est de 1,32 bulles/mrem avec un écart-type de 0,10 bulles/mrem.

\section{Recommandations d'emploi}

Les recommandations ci-après s'imposent :

1) conserver les dosimètres dans leur emballage dans une pièce où la température varie peu, de quelques degrés autour de $20^{\circ} \mathrm{C}$, il est surtout déconseillé de les soumettre à des chocs thermiques ;

2) effectuer un essai de réception et de dispersion sur un petit nombre, pris au hasard, du lot livré, il a été constaté un écart maximal faible, $\pm 10 \%$, entre la sensibilité mesurée et celle du constructeur, pour chaque détecteur ;

3) acheter le lecteur "automatique" de dosimètres, mais il faut un opérateur qui corrige la lecture "à la main", pour la rendre plus juste ;

4) coupler le dosimètre BD 100R-PND avec un dosimètre BDT, pour surveiller l'irradiation des agents dans les champs de neutrons ralentis;

5) contrôler périodiquement, une fois par mois, la sensibilité du dosimètre à l'aide d'une source Am-Be (voir $\S 4.2$ ) (il faut rappeler qu'en utilisation journalière comportant un cycle de décompression et recompression la durée de vie du détecteur est de l'ordre de 3 mois) ;

6) si un dosimètre a subi une forte augmentation ou une forte diminution de température par rapport à $20^{\circ} \mathrm{C}$, contrôler sa sensibilité avec la source Am$\mathrm{Be}$ avant de l'affecter à l'agent; il faut signaler qu'après retour à $20^{\circ} \mathrm{C}$, après avoir subi des températures de $+15^{\circ} \mathrm{C}$ et de $+40^{\circ} \mathrm{C}$, la perte maximale de sensibilité est de l'ordre de $15 \%$. 


\section{RÉFÉRENCES}

[1] CENTRE TECHNIQUE D'HOMOLOGATION DE L'INSTRUMENTATION DE RADIOPROTECTION (CTHIR). - Dosimètres neutrons à bulles types BD $100 \mathrm{R}$ PND et BDT. Rapport d'essais CTHIR/94-101, 1994.

[2] COMMISSION ELECTROTECHNIQUE INTERNATIONALE (CEI). Moniteurs personnels à lecture directe d'équivalent de dose et/ou de débit d'équivalent de dose pour les rayonnements $X$, gamma, bêta d'énergie élevée et de neutrons. CEI-45-B (S) 120, 1993.

[3] ETATS-UNIS. NATIONAL COUNCIL ON RADIATION PROTECTION AND MEASUREMENTS (NCRP). - Protection against neutron radiation (NCRP report 38). Washington : NCRP, 1971.

[4] INTERNATIONAL COMMISSION ON RADIOLOGICAL PROTECTION (ICRP). - Data for protection against ionizing radiation from external sources, supplement to ICRP Publication 15 (ICRP Publication 21). Oxford : Pergamon press, 1973.

[5] INTERNATIONAL COMMISSION ON RADIOLOGICAL UNITS AND MEASUREMENTS (ICRU). - Determination of dose equivalents resulting from external radiation sources (ICRU report 39). Bethesda : ICRU, 1992.

[6] INTERNATIONAL COMMISSION ON RADIOLOGICAL UNITS AND MEASUREMENTS (ICRU). - Measurements of dose equivalents from external photon and electron radiations (ICRU report 47). Bethesda : ICRU, 1992.

[7] INTERNATIONAL STANDARDS ORGANISATION (ISO). - Rayonnements neutroniques de référence destinés à l'étalonnage des instruments de mesure des neutrons utilisés en radioprotection et à la détermination de leur réponse en fonction de l'énergie des neutrons (ISO 8529). Paris : AFNOR, 1989. 